\title{
Why do certain limacons have a dimple
}

H. Azad and M. T. Mustafa

Submitted September 2005; accepted December 2005

\section{Introduction}

The graphing of Cartesian curves is analysed in great detail in all the standard textbooks, whereas polar curves are given a very careless treatment. Is there a calculus for polar and in general parametric curves? Why do certain limacons have a dimple? How were such questions handled before our dependence on technology? The answers to such questions are given here, through intuitive and geometric arguments.

All the standard text books of calculus, for instance (1-3), treat the graphing of curves in rectangular co-ordinates with great care. For example, for graphs of functions like $y=x^{3}$ or $y=x+x^{2}$, the critical points of the function, its points of inflection and its behaviour at infinity are determined for a good sketch. However, when it comes to sketching polar curves in more advanced courses, the attitude changes dramatically. Here is the advice which is given by Anton et al. (1, p. 726):

'The most elementary way to graph the polar equation $r=f(\theta)$ is to plot points. The idea is to choose some typical values of $\theta$, calculate the corresponding value of $r$ and plot the point $(r, \theta)$ in polar coordinates.'

The books (2-4) give similar advice.

In lower level calculus courses, the student is supposed to work with epsilons and deltas and to think analytically, whereas in higher level courses, when dealing with polar curves, he is asked to do just the opposite. For instance, for certain families of polar curves like the limacons, the student is asked to memorize their shapes, without any discussion or a hint as to how their shapes could be worked out [see e.g. (2, p. 571)]. Since the hall-mark of the Calculus Reform Movement is focus on understanding, there should be an alternative. Here we present one alternative.

\section{Some principles for sketching polar curves}

The graphing of polar equations can be based on three principles:

(1) If $r=f(\theta)$ and $f\left(\theta_{0}\right)=0$, then the ray $\theta=\theta_{0}$ is tangent at the origin to the graph of $r=f(\theta)$ in the $(X, Y)$-plane.

(2) The point $(-r, \theta)$ is obtained from $(r, \theta)$ by rotating it by $180^{\circ}$.

(3) If $\mathrm{d} y / \mathrm{d} x \neq 0$ at $(a, b)$, then along the curve and near $(a, b), x$ can be solved as a function of $y$. 
Regarding (1) and (3) the students are completely satisfied by pictorial arguments like the following:

- For (1), take a curve passing through origin $\mathrm{O}$, a point $\mathrm{P}$ on the curve and a ray OP. As the point $\mathrm{P}$ moves on the curve towards $\mathrm{O}$, the ray $\mathrm{OP}$ approaches the ray $\theta=\theta_{0}$ which is tangent to the curve at the origin.

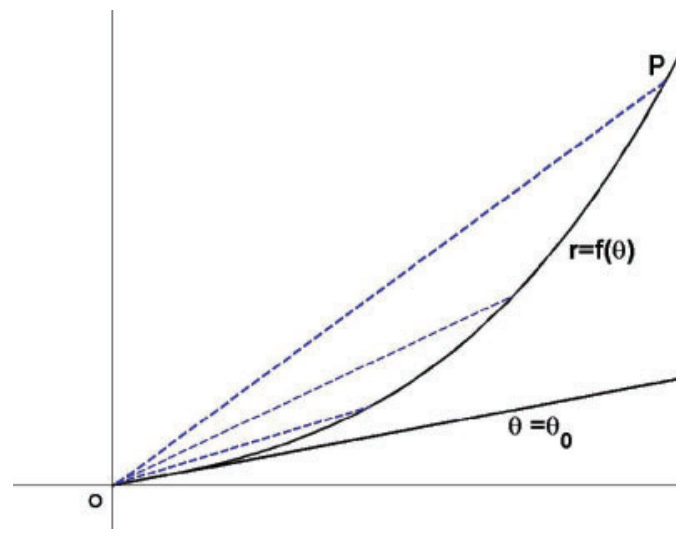

- For (3), if the tangent line is not horizontal, then near the point, projecting on the $y$-axis gives $x$ as a function of $y$.

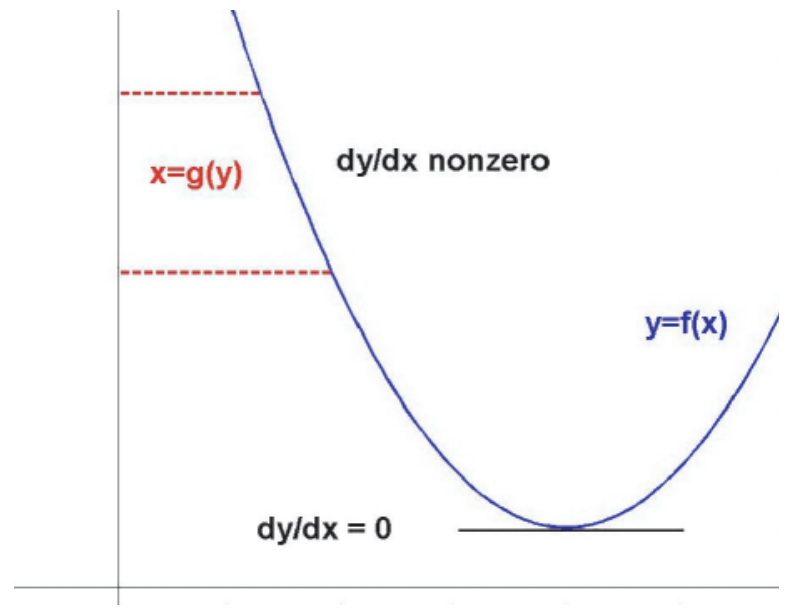

The third principle is needed if one wants to determine changes in shape of the curve (i.e. changes in curvature).

\section{Illustration of graphing using these principles}

Example 1: Let us see how these ideas help in explaining why the curve $r=\sin \theta-(1 / 2)$ has two loops, one inside the other. 
Steps:

(1) Find theta where $r=0$. This will determine intervals where $r$ is positive and where it is negative, and tangent rays at the origin. The values of $\theta$ are $\theta=\pi / 6$ and $\theta=5 \pi / 6$. In the interval $\pi / 6 \leq \theta \leq 5 \pi / 6, r \geq 0$.

(2) Draw the part of the curve where $r \geq 0$. The rays $\theta=\pi / 6, \theta=5 \pi / 6$ are tangent at the origin. Since, now $r$ is the distance of the moving point from the origin, it is easy to plot the points $r=f(\theta)$ by hand and we analyse its behaviour as for curves in rectangular coordinates. For this, we find where $r$ is increasing and where it is decreasing. The smallest value of $r$ is 0 and the largest value is $1 / 2$. So the graph of this part appears like this:

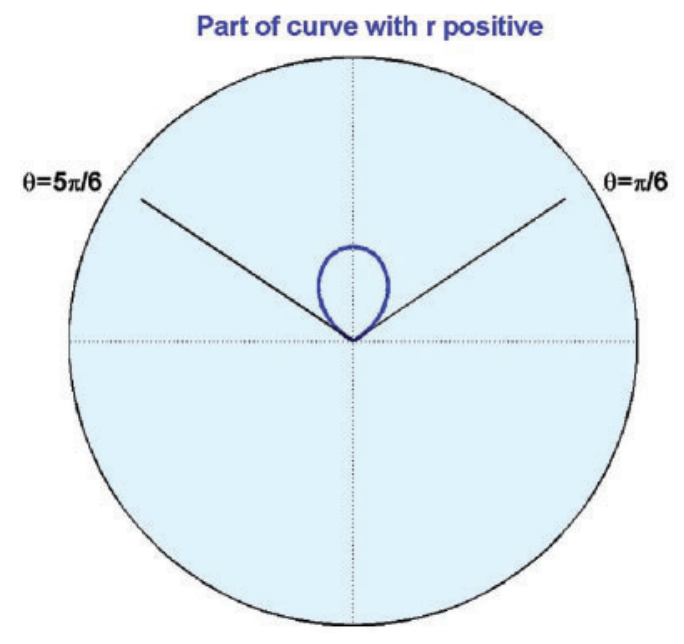

(3) Draw the part of the curve where $r \leq 0$. First draw the curve $r=-(\sin \theta-(1 / 2))$ in the interval $5 \pi / 6$ to $2 \pi+\pi / 6$. The smallest value of $r$ is 0 and largest value is $3 / 2$. The rays $\theta=5 \pi / 6, \quad \theta=2 \pi+\pi / 6 \equiv \pi / 6$ are tangent at $\mathrm{O}$.

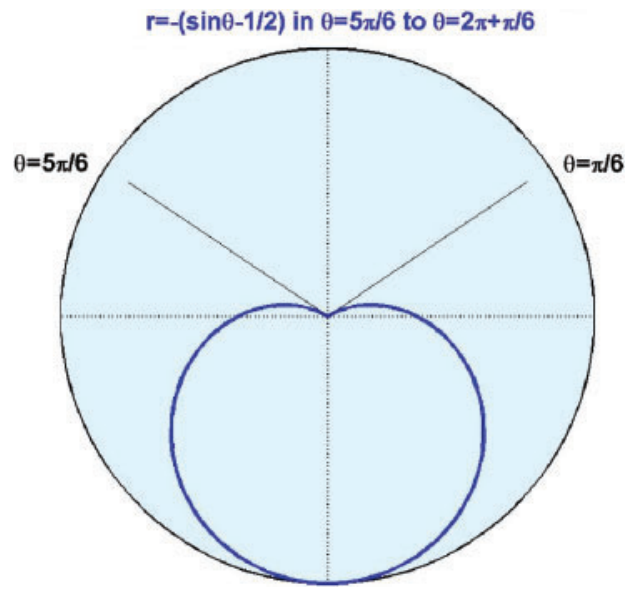


Now rotate this through $180^{\circ}$. After rotation the smaller loop comes inside this bigger loop and the bigger loop is tangent to the rays $\theta=7 \pi / 6, \theta=11 \pi / 6$. This gives the complete curve.

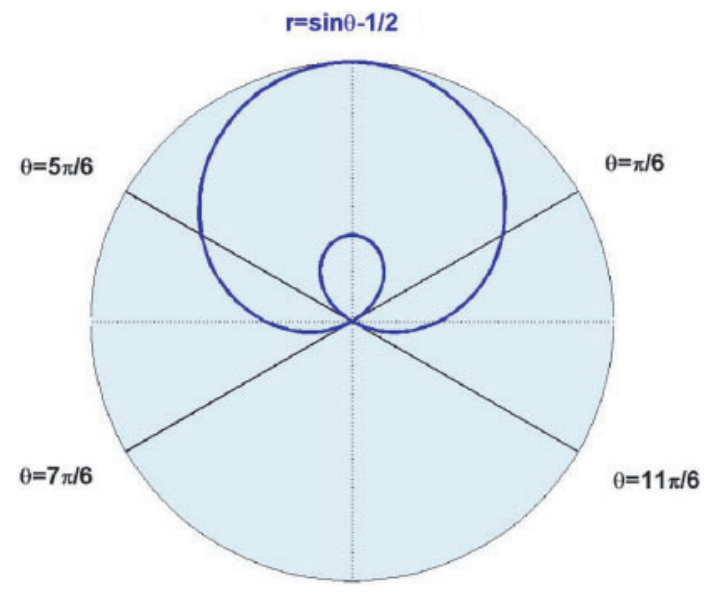

\section{Example 2: Roses}

The graphs for roses, etc., are simpler to draw, with the tangent rays at the origin separating neatly where $r=f(\theta)$ is positive and where it is negative. This also helps the student to see why for example, for the curve $r=\sin (2 \theta)$ there are four loops, whereas for $r=\sin (3 \theta)$ the curve is traced twice and there are only three loops.

\section{Why do certain limacons have a dimple?}

These are the curves $r=\mathrm{a}+b \sin \theta$ (and $r=\mathrm{a}+b \cos \theta$ ), with $a b \neq 0$. The books (1-3) give the following criterion: If $1<|a / b|<2$ then the curve has a dimple.

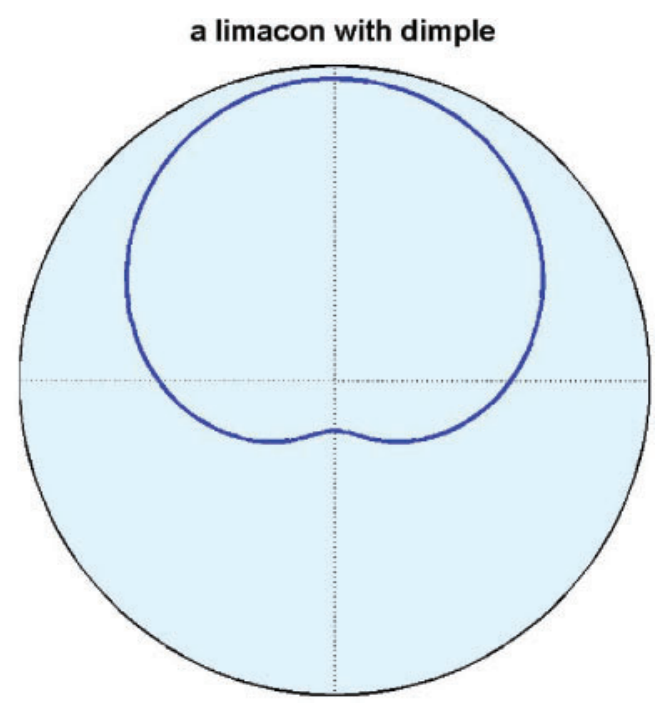


What is the explanation for this? This is due to changes in concavity as we move along the curve, but this needs to be interpreted properly. Is the circle convex or concave or neither? A satisfactory treatment of this simple question is given in the next section. However, an explanation along the following lines is quite sufficient in the class room. At points where $\mathrm{d} x / \mathrm{d} \theta \neq 0, \theta$ can be solved as a function of $x$ and therefore $y$ itself is a function of $x$. So it makes sense to compute $\mathrm{d}^{2} y / \mathrm{d} x^{2}$ and to determine the possible points of inflections.

A computation shows that where $\mathrm{d} x / \mathrm{d} \theta \neq 0, \mathrm{~d}^{2} y / \mathrm{d} x^{2}=0$ if and only if $\lambda^{2}+3 \lambda \sin \theta+2=0$.

This means that

$$
\sin \theta=\frac{-\left(2+\lambda^{2}\right)}{3 \lambda}
$$

Taking absolute values this gives,

$$
(|\lambda|-1)(|\lambda|-2) \leq 0
$$

So,

$$
1 \leq|\lambda| \leq 2
$$

and the values of $\theta$ are given by equation [1]. It remains to be checked whether these possible points of inflection are indeed points of inflection. This follows from one of the exercises given in the end. At points where $\mathrm{d} x / \mathrm{d} \theta=0$, we have $\mathrm{d} y / \mathrm{d} \theta \neq 0$ and similar computations give the same result.

\section{Using curvature to determine dimple of limacon}

Although this treatment is sufficient for the class room, it is still not quite satisfactory because we have to deal separately with the cases $\mathrm{d} x / \mathrm{d} \theta \neq 0$ and $\mathrm{d} y / \mathrm{d} \theta \neq 0$. The reader who has done these complete computations will see a repetition of arguments, which is not satisfactory. A satisfactory treatment is given by considering the curvature of plane curves where the $X$ and $Y$ co-ordinates are treated on an equal footing.

What is curvature? Intuitively, the curvature of a plane curve is the rate at which the curve is turning or changing direction. Since the tangent vector gives the direction of the curve, it is clear that the curvature of a plane curve can be described as the rate at which the tangent vector is turning. In more detail:

- take a parameterized curve (with parameter $t$ ) in the plane and a fixed reference direction (say $X$-axis) (Fig. 1a).

- take a point $p$ on the curve and unit tangent vector at $p$. As $t$ changes, the unit tangent vector moves along the curve. We measure the angle of rotation $\theta(t)$ from the initial position (Fig. 1b). The rate of change of this angle of rotation with respect to arc length is the signed curvature of the curve.

In the following figures, $\theta(t)$ is the counter clockwise angle of rotation from the fixed reference direction to the direction of the tangent vector along the curve.

A formula for the curvature is easily obtained. If $\gamma(t)=(x(t), y(t))$ is a parameterized curve, then the unit tangent vector along the curve is given by $\dot{\gamma}(t) /|\dot{\gamma}(t)|$. If the components of this unit vector are $(f(t), g(t))$ then the curvature is given by $\left(f g^{\prime}-g f^{\prime}\right) /\left|\gamma^{\prime}(t)\right|$. So, the sign of curvature 
(a)

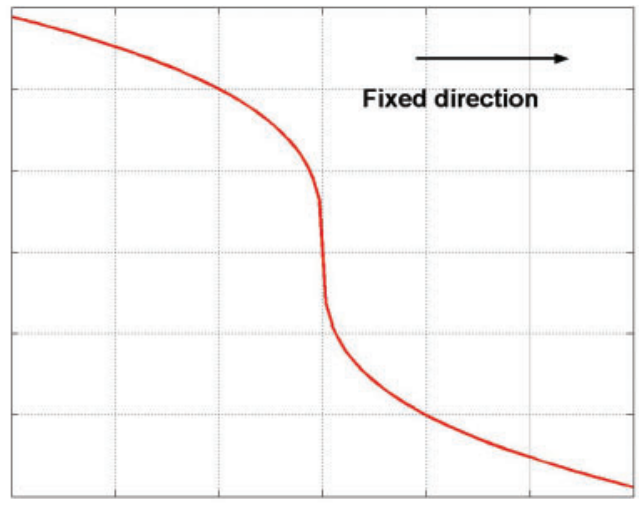

(b)

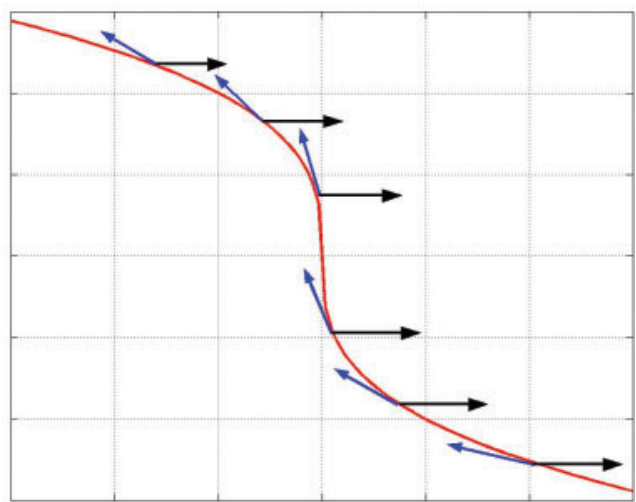

Fig 1. Signed curvature.

is given by the sign of $\left(f g^{\prime}-g f^{\prime}\right)$. Computations simplify considerably by using the following observation: the sign of $(h / k)(l / k)^{\prime}-(l / k)(h / k)^{\prime}$ is the same as the sign of $h l^{\prime}-l h^{\prime}$, for any functions $h, k$ and $l$ for which these expressions are defined. The following examples show that positive and negative curvature have no geometric meaning; it is only the change in sign of curvature which has a geometric meaning.

Example: Consider the circle $f(t)=(R \cos (t), R \sin (t))$. As the reference direction we take the unit tangent vector [1,0]. The unit tangent vector for the parameter value $t$ is $(-\sin (t), \cos (t))$ which is equal to $(\cos (t+\pi / 2), \sin (t+\pi / 2))$. So here $\theta(t)=t+\pi / 2$. As $\mathrm{d} s / \mathrm{d} t=R$, where $s$ is the arc length function along the curve, we see that $\mathrm{d} \theta / \mathrm{d} s=1 / R$.

Similarly, if we move along the circle in clockwise direction, the curvature will be $-1 / R$. So there is no change in sign of curvature as we move along the circle in a fixed orientation.

Curvature $=1 / R$

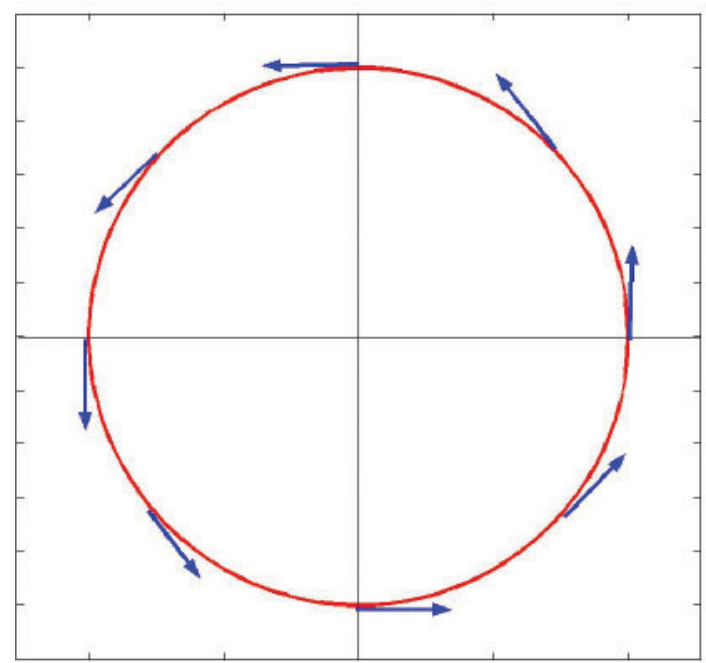

Reference direction
Curvature $=-1 / R$

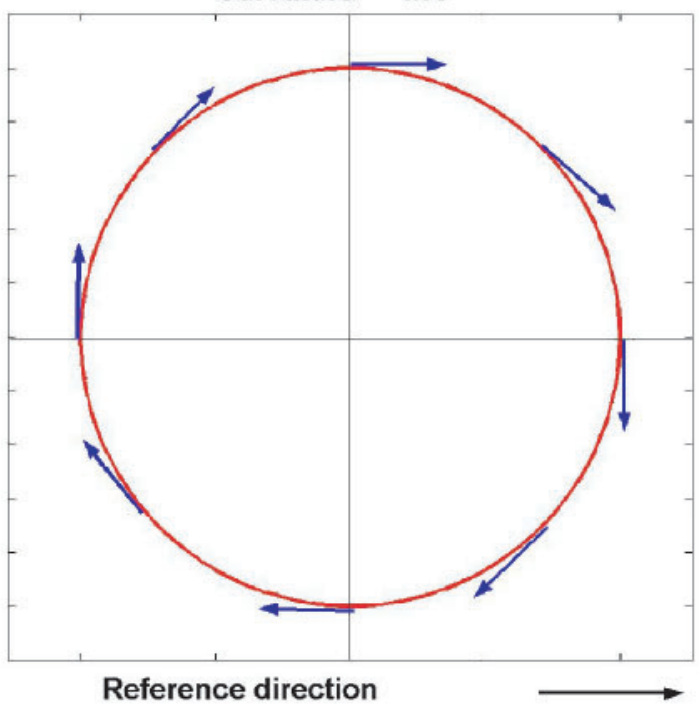


Example: The following figures show the relationship between points of inflection and change of curvature.

- $y=x^{2}$ with parameterization $\gamma(t)=\left(t, t^{2}\right)$. As we move along the curve, $\theta(t)$ increases, hence curvature is positive and there are no points of inflection.

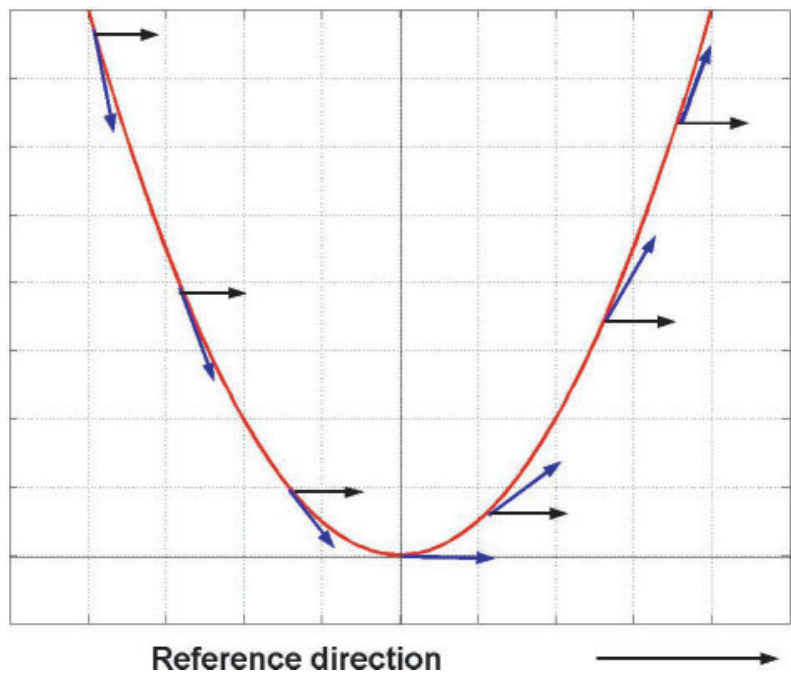

- $y=x^{2}$ with parameterization $\gamma(t)=\left(-t, t^{2}\right)$. As we move along the curve, $\theta(t)$ decreases, hence curvature is negative and there are no points of inflection.

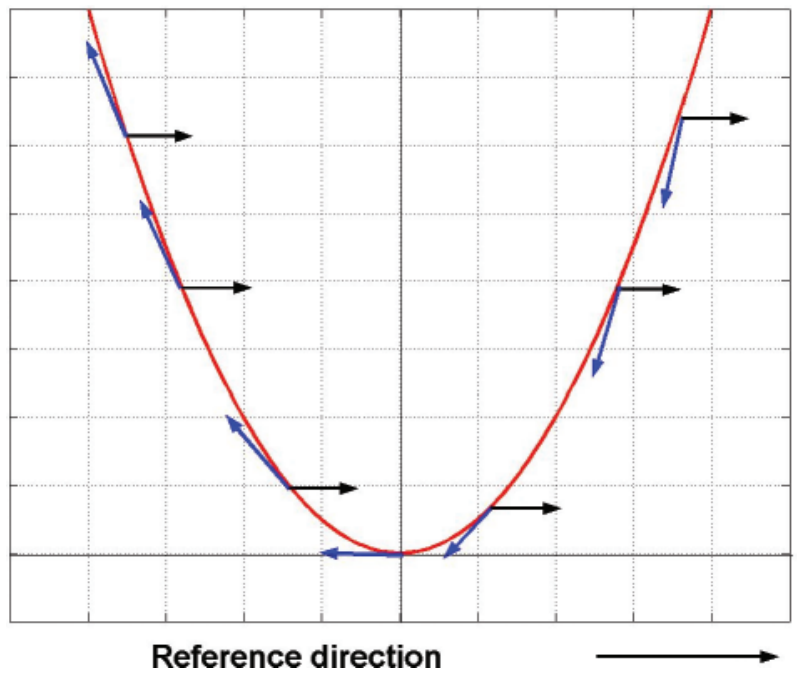

- $y=x^{3}$ with parameterization $\gamma(t)=\left(t, t^{3}\right)$. As we move along the curve from the 3 rd quadrant to the 1st quadrant, $\theta(t)$ decreases up to the origin and increases thereafter. The curvature changes from negative to positive at origin, which is the point of inflection. 


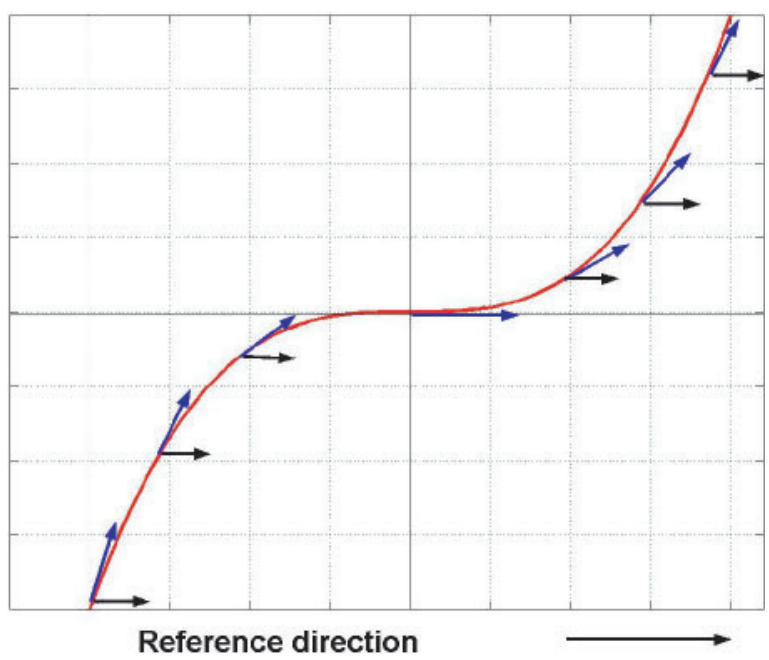

- $y=x^{3}$ with parameterization $\gamma(t)=\left(-t, t^{3}\right)$. As we move along the curve from the 1 st quadrant to the 3rd quadrant, $\theta(t)$ decreases up to the origin and increases thereafter. The curvature changes from negative to positive at origin, which is the point of inflection.

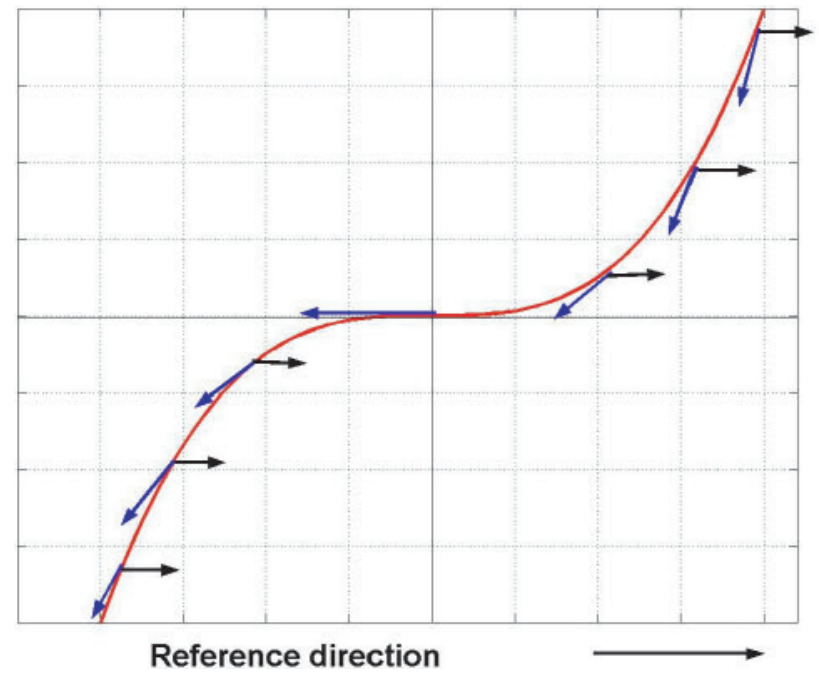

\section{Limacons from the point of view of curvature}

Consider the curve $r(t)=a+b \sin (t)$ in the XY plane. Since scaling does not change the shape of the curve we may assume that $r(t)=\lambda+\sin (t)$. The curve is singular if for some value of $t$, $\mathrm{d} x / \mathrm{d} t=0$ and $\mathrm{d} y / \mathrm{d} t=0$. So this curve is non-singular if $|\lambda| \neq 1$. A computation shows that the sign of curvature is given by the sign of $\lambda^{2}+3 \lambda \sin t+2$. The equation $\lambda^{2}+3 \lambda \sin t+2=0$ gives the inequality $1 \leq|\lambda| \leq 2$ as in equation [2]. This gives two values for $t$ and there is an actual change of 
curvature as we pass through these values of $t$ (see suggested exercises). It is this change in curvature at two values of $t$ which accounts for the dimple in the corresponding limacons.

\section{Exercises and concluding remarks}

When we draw polar curves, we are actually looking at the image of the curve $r=f(\theta)$ in the $(r, \theta)$ plane under the map $(r, \theta) \rightarrow(r \cos \theta, r \sin \theta)$. Even if the original curve has well-defined tangent lines, the image curve may acquire several tangents at the same point and still be non singular in the sense that $\left(x^{\prime}(\theta), y^{\prime}(\theta)\right) \neq 0$ for any $\theta$. If $\left(x^{\prime}(\theta), y^{\prime}(\theta)\right)=0$ for some $\theta$, we call the curve singular.

Exercise

Verify that if $\lambda^{2}+3 \lambda \sin t+2=0$ for some $t$ and $1<|\lambda|<2$, then the curvature changes as we pass through $t$ (use Taylor series of appropriate order for $h(t+\varepsilon)$. If $|\lambda|=1$, then the curve has a singular point. If $|\lambda|=2$, then the curve is non-singular and there is no change in curvature.

In elementary calculus, we deal with tangent lines, arc length and concavity of curves. The analogue for this for parametric curves have obvious meaning with the exception that concavity and convexity have no geometric meaning-only change in sign of curvature has a geometric meaning. The calculus of parameterized curves is the differential geometry of curves, see e.g. (5).

Finally, we should add that this note is not meant to be a criticism of technology. In fact, all the graphics were done with Matlab. The message which we want to convey is that technology and conceptual understanding enrich and enhance each other and with the wide availability of advanced software and the consequent freedom from drudgery, our undergraduates are bound to ask increasingly more conceptual questions.

\section{References}

1. Anton, H., Bivens, I. and Davis, S. (2002) Calculus, 7th edn, John Wiley \& Sons, Inc.

2. Stewart, J. (1995) Calculus, 3rd edn, Brooks/Cole Publishing Company.

3. Finney, R. L., Weir M. D. and Giordano, F. R. (2003) Thomas' Calculus, 10th edn, Addison Wesley.

4. Johnson, R. F. and Kiokemeister, F. L. (1969) Calculus with Analytic Geometry, 4th edn, Allyn and Bacon, Inc., Boston, USA.

5. O’Neill, B. (1997) Elementary Differential Geometry, 2nd edn, Academic Press, New York, USA.

Address for correspondence: H. Azad, Department of Mathematical Sciences, King Fahd University of Petroleum and Minerals, Dhahran 31261, Saudi Arabia. E-mail: hassanaz@kfupm.edu.sa

H. Azad is Professor of Mathematics at the King Fahd University in Dhahran, Saudi Arabia. He received his Ph.D. in 1977 from the University of Notre Dame, USA.

M. T. Mustafa is Assistant Professor of Mathematics at the King Fahd University in Dhahran, Saudi Arabia. He received his Ph.D. in 1996 from the University of Leeds, U.K. 